The BMJ

Cite this as: BMJ2020;370:m3757 http://dx.doi.org/10.1136/bmj.m3757 Published: 28 September 2020
NEWS ANALYSIS

\section{Covid-19: Government poised to amend regulations to allow use of unlicensed vaccine}

\author{
Experts say robust legal protections are needed to inspire public confidence. Clare Dyer reports
}

Clare Dyer

The UK government has set out plans to amend drug regulations in case it decides that covid-19 vaccines should be used before they are licensed, in a bid to roll them out more quickly. ${ }^{1}$

In a consultation on the proposals that ran from 28 August to 18 September the Department of Health and Social Care for England explained that if a suitable vaccine emerged with strong evidence of safety, quality, and efficacy the government would seek to license it through the usual route but could supply it in the meantime.

\section{"Unlicensed" not "untested"}

The consultation document said that if there were a "compelling case, on public health grounds, for using a vaccine before it is given a product licence, given the nature of the threat we face, the Joint Committee on Vaccination and Immunisation may take the very unusual step of advising the UK government to use a tested, unlicensed vaccine against covid-19, and we need to make sure that the right legislative measures are in place to deal with that scenario.”

The document added, "A covid-19 vaccine would only be authorised in this way if the UK's licensing authority was satisfied that there is sufficient evidence to demonstrate the safety, quality, and efficacy of the vaccine. 'Unlicensed' does not mean 'untested."

The consultation, and the timeframe in which it was conducted, prompted some people to post their concerns on social media. A post on a local residents' Facebook group in Devon reads, “After 18 Sept, the government are going to say they consulted the public and because there were no objections, we all want and consent to the rollout of unlicensed vaccines, and that we are happy for non-medical staff to administer them and happy to accept we will not have the ability to ask for compensation if we face damage to our health."2

The Human Medicine Regulations 2012 already allow the licensing authority to temporarily authorise the supply of an unlicensed product in response to certain public health threats, including the suspected spread of pathogens. The proposed change would allow conditions to be attached "to ensure product safety, quality, and efficacy"

\section{Extending immunity from being sued}

The 2012 regulations also give healthcare professionals and manufacturers immunity from being sued in the civil courts for the use of some unlicensed products recommended by the licensing authority in response to a public health threat. The new regulations would extend the immunity to drug companies that have not manufactured the product but placed it on the market with the approval of the licensing authority, and they clarify the consequences for a breach of conditions imposed by the authority.

Immunity would be lost in the case of a "serious breach," and the Consumer Protection Act 1987 would still apply, making manufacturers liable if the product were defective.

Further changes would allow an expanded range of professionals, including midwives, paramedics, and physiotherapists, to be trained to help deliver a mass covid-19 and an upscaled flu vaccination programme. These professionals would also have immunity from civil liability.

Stephen Evans, professor of pharmacoepidemiology at the London School of Hygiene and Tropical Medicine, said that expanding the vaccination workforce was "eminently sensible and should have been planned for many months ago." He added, "It is clear that the current system of administration would not be capable of vaccinating almost the entire population in a short time, so this could be very sensible, provided the training of the new groups to carry out vaccinations is good and is perceived by the general public to be sufficient training."

\section{Vaccine hesitancy}

Social media posts play into existing concerns that many people might not accept the vaccine, as surveys indicate.

Lawyers have told the Department for Health and Social Care that to inspire public confidence it must provide redress for the few people who might experience adverse effects.

Bozena Michalowska, a partner specialising in product liability at the law firm Leigh Day, said, "I do not believe that people will want to play Russian roulette with their health by taking a vaccine which they know nothing about, especially when they know that the risks they take are just taken by them and not a shared risk and they will not have sufficient protection should things go wrong."

Peter Todd, a partner specialising in vaccine injury claims at solicitors Hodge Jones and Allen, said, "The fact that there has never yet been a successful vaccine against a coronavirus, coupled with some proposed covid-19 vaccines having novel mechanisms of 
efficacy and a very short period of clinical testing, means that there could be some additional risks in these circumstances which may put people off, especially in the light of civil immunity.

"A proper compensation programme may be a cheap and straightforward solution to neutralising vaccine hesitancy and bring the pandemic to an early end.”

1 Department of Health and Social Care. Consultation document: Changes to Human Medicine Regulations to support the rollout of COVID-19 vaccines. 28 Aug 2020

https://www.gov.uk/government/consultations/distributing-vaccines-and-treatments-for-covid-

19-and-flu/consultation-document-changes-to-human-medicine-regulations-to-support-the-rolloutof-covid-19-vaccines.

2 https://www.facebook.com/groups/319841736731/permalink/10157648614676732. 